\title{
CONTROL BACKSTEPPING DE UN ROBOT SCARA CON INCERTIDUMBRE PARAMÉTRICA
}

\author{
A BACKSTEPPING CONTROL FOR SCARA ROBOT BASED ON PARAMETRIC UNCERTAINTY \\ Víctor Hugo Mosquera Leyton \\ M.Sc. en Automática, Universidad del Cauca, Profesor Titular, \\ Departamento de Electrónica, Instrumentación y Control. Universidad del Cauca. \\ mosquera@unicauca.edu.co \\ Óscar Andrés Vivas Albán \\ Dr. en Robótica, Université Montpellier II, Francia, Profesor Titular, \\ Departamento de Electrónica, Instrumentación y Control. Universidad del Cauca. \\ avivas@unicauca.edu.co
}

Fecha de recepción: 15 de septiembre de 2011

Fecha de aprobación: 3 de mayo de 2012

\section{RESUMEN}

Uno de los principales problemas en el control por modelo de referencia es que las dinámicas no modeladas, la incertidumbre paramétrica y el acoplamiento dinámico, afectan el desempeño del sistema de control. Este artículo presenta una estrategia de control bajo el enfoque backstepping, con el fin de garantizar en primera instancia la estabilidad en lazo cerrado del sistema de control de un manipulador SCARA de $4^{\circ}$ de libertad, y en segunda instancia generar un sistema que sea robusto ante incertidumbres paramétricas, sin presentar grandes esfuerzos de control.

Palabras clave: control backstepping, control de robots, control no lineal, control robusto, incertidumbre paramétrica.

\begin{abstract}
One of the typical problems of model-based control strategies is impact on control system performance due to non-modeled dynamics, parametric uncertainty and dynamic coupling. This paper offers a control strategy under a back stepping approach in order to ensure stability into a closed loop of a 4-DOF SCARA manipulator, and also to generate a system with robustness against parametric uncertainties, without increase control efforts.
\end{abstract}

Keywords: back stepping control, robot control, nonlinear control, robust control, parametric uncertainty. 


\section{INTRODUCCIÓN}

Los robots manipuladores que se utilizan en procesos manufactureros requieren por lo general, que el efector final se desplace, siguiendo una trayectoria deseada y a una determinada velocidad. Para lograrlo, se recurre a estrategias de control clásico tipo PID, que no son muy precisas a altas velocidades o bien, a controles por par calculado, los cuales presentan bajo desempeño en presencia de incertidumbre en los parámetros del manipulador, lo cual significa que sus parámetros dinámicos no son conocidos con exactitud [1].

Las estrategias de control robusto se desarrollan con el fin de estabilizar sistemas que presentan errores en el modelo y dinámicas no modeladas. Los modelos con incertidumbre están presentes con frecuencia, en la robótica donde dichas incertidumbres se deben a cambios en la carga del robot, simplificación del modelo dinámico y fuerzas de fricción, entre otras. La incertidumbre hace que los parámetros de desempeño del sistema y el error de seguimiento se vean afectados [1-3], por tal razón, es importante el diseñar esquemas de control robusto, teniendo en cuenta además, la no linealidad de la dinámica de los manipuladores. Al respecto se han realizados varios trabajos con el fin de aplicar estrategias de control adaptativo y óptimo [2-5], los cuales parten de la propuesta de Spong [6] para minimizar los efectos de la incertidumbre en el desempeño del seguimiento de un manipulador de $2^{\circ}$ de libertad, pero en estos trabajos no se tiene en cuenta los esfuerzos de control, parámetro muy importante para implementar el controlador, debido a las limitaciones presentes en los actuadores del manipulador. Backstepping es un procedimiento recursivo que combina la elección de una función de Lyapunov con el diseño de un control por realimentación [7-8]. Este procedimiento descompone el problema original en una secuencia de problemas de diseño para sistemas de orden reducido (que pueden llegar a ser escalares). Explotando la flexibilidad adicional que existe con sistemas de bajo orden y escalares, backstepping puede resolver a menudo problemas de estabilización, seguimiento y control robusto bajo condiciones menos restrictivas que las encontradas en otros métodos [7]. Algunos autores han aplicado esta estrategia de control en el seguimiento de trayectorias, control de sistemas neumáticos, de vehículos no tripulados y de motores, obteniendo muy buenos resultados [812]. Por tal motivo, este trabajo busca una ley de control robusta eficiente con respecto de los esfuerzos de control en el caso de la incertidumbre paramétrica.

Este artículo presenta la aplicación de la técnica de control backstepping a un manipulador tipo SCARA de $4^{\circ}$ de libertad. En la sección 1 se presenta el modelo dinámico del manipulador con presencia de incertidumbres; la estrategia de control backstepping y la estrategia de control robusto se explican en las secciones 2 y 4 respectivamente; la sección 4 presenta las características del robot SCARA; la sección 5 presenta el control por par calculado (CTC), para el robot SCARA; la sección 6 muestra los resultados de simulación, y finalmente, las conclusiones del trabajo se presentan en la sección 7. 


\section{MODELO DINÁMICO DEL ROBOT}

Como se conoce, el modelo dinámico de un manipulador relaciona los pares con las posiciones, velocidades y aceleraciones de las articulaciones que lo componen. La Ec. (1) muestra la representación matemática de este modelo [13].

$$
\Gamma=M(q) \ddot{q}+C(q, \dot{q}) \dot{q}+Q(q)
$$

En la anterior ecuación, $\Gamma$ representa el vector de par de las articulaciones, $M$ la matriz de inercia, $C$ el vector de fuerzas centrífugas y de Coriolis, $Q$ el vector de fuerzas de gravedad yq, $\dot{\boldsymbol{q}}$ y $\ddot{\boldsymbol{q}}$ los vectores de posiciones, velocidades y aceleraciones de las articulaciones respectivamente. Se asume incertidumbre en las matrices de inercia $(M)$ y de fuerzas centrífugas y de Coriolis $(C)$, lo cual significa que los parámetros dinámicos presentes no son conocidos con exactitud o que hay dinámicas que no han sido modeladas. Las Ecs. (2) y (3) muestran las matrices $M$ y $C$, compuestas por $M_{0}$ y $C_{0}$ que representan el modelo nominal o real del robot y de $\boldsymbol{\Delta} \boldsymbol{M}$ y $\boldsymbol{\Delta} \boldsymbol{C}$ que indican las incertidumbres del modelo.

$$
\begin{aligned}
& M=M_{0}+\Delta M \\
& C=C_{0}+\Delta C
\end{aligned}
$$

Sustituyendo las Ecs. (2) y (3) en la Ec. (1) se obtiene:

$$
M_{0}(\boldsymbol{q}) \ddot{q}+C_{0}(q, \dot{q}) \dot{q}+G=\Gamma+\Delta
$$

Donde $\boldsymbol{\Delta}$ representa la incertidumbre y las dinámicas no modeladas del robot.

\section{LEY DE CONTROL BACKSTEPPING CON INCERTIDUMBRE}

Backstepping puede resolver problemas de estabilización, seguimiento y control robusto bajo condiciones menos restrictivas que las encontradas en otros métodos [7]. Con esta estrategia de control, se busca tener un error de seguimiento a una trayectoria deseada muy pequeño, pese a la presencia de incertidumbres paramétricas, y sin generar grandes esfuerzos de control.

El procedimiento que se presenta a continuación, busca obtener otra representación del modelo dinámico inverso del manipulador, con el fin de obtener una señal de control virtual $(\dot{\xi})$ y otra real (u). Ésta última será la integral de la señal virtual de control que estabilizará el sistema. La forma general que se utiliza para aplicar el control backstepping es:

$$
\begin{gathered}
\dot{x}=f(x)+ \\
g(x) \xi, f(0)=0 \\
\dot{\xi}=u
\end{gathered}
$$


Como se mencionó, el diseño de la estrategia de control backsteping con incertidumbre paramétrica parte de la determinación de las variables que definirán la señal de control virtual del sistema. Dicha señal de control virtual será el vector de error de posición (Ec. 7). Las Ecs. (7) y (8) presentan respectivamente, los vectores de error de posición y velocidad de las articulaciones del robot.

$$
\begin{aligned}
& x_{1}=\tilde{\boldsymbol{q}}=\boldsymbol{q}-\boldsymbol{q}_{d} \\
& \dot{\boldsymbol{x}}_{1}=\dot{\tilde{\boldsymbol{q}}}=\dot{\boldsymbol{q}}-\dot{\boldsymbol{q}}_{d}
\end{aligned}
$$

Donde $\boldsymbol{q}_{\boldsymbol{d}}$ y $\dot{\boldsymbol{q}}_{\boldsymbol{d}}$ son la posición y velocidad deseadas respectivamente. Siguiendo la metodología de Spong, Zhu y Burkan [6, 3], se define el vector de corrección de velocidad como:

$$
x_{2}=\dot{x}_{1}-\sigma=\dot{x}_{1}+K x_{1}
$$

Donde $K$ es una matriz diagonal y definida positiva, para obtener una combinación lineal entre $x_{1}$, $x_{2} y \dot{\boldsymbol{x}}_{1}$. Al reàlizar un sencillo proceso aritmético sobre la Ec. (9), se obtiene:

$$
\dot{x}_{1}=x_{2}-K x_{1}
$$

Derivando la Ec. 8 queda: $\ddot{\boldsymbol{x}}_{1}=\ddot{\boldsymbol{q}}-\ddot{\boldsymbol{q}}_{d}$

Sustituyendo $\ddot{\boldsymbol{x}}_{1}$ en la derivada de la Ec. 9, se obtiene:

$$
\dot{\boldsymbol{x}}_{2}=\ddot{\boldsymbol{x}}_{1}+K \dot{\boldsymbol{x}}_{1}=\ddot{\boldsymbol{q}}-\ddot{\boldsymbol{q}}_{d}+K \dot{\boldsymbol{x}}_{1}
$$

Despejando $\ddot{\boldsymbol{q}}$ de la Ec. (4) y sustituyéndolo en la Ec. (11), se obtiene:

$$
\begin{aligned}
& \dot{x}_{2}=M_{0}^{-1}\left(\Gamma+\Delta-C_{0} \dot{q}-G\right)-\ddot{q}_{d}+K x_{2}-K^{2} x_{1} \\
& \dot{x}_{1}=-K x_{1}+x_{2}
\end{aligned}
$$

De las Ecs. (12) y (13) se puede observar con claridad que se logra un modelo, cuya entrada virtual de control es $\boldsymbol{x}_{\boldsymbol{1}}$ y cuya integral representa la señal de control real del sistema, típico de un control backstepping con incertidumbre paramétrica [7].

Para obtener la ley de control que estabilice el sistema representado por las Ec. (12) y (13), se selecciona en primera instancia la función de Lyapunov $V_{a}$ con la cual se espera demostrar que el sistema es asintóticamente estable. Esta ecuación es:

$$
\mathrm{V}_{\mathrm{a}}=\frac{1}{2} x_{2}^{T} M_{0} x_{2}+\frac{1}{2} x_{1}^{T} K x_{1}
$$


Derivando la función de Lyapunov [7], y sustituyendo las Ecs. (12) y (13) se obtiene:

$$
\begin{aligned}
\dot{V}_{\mathrm{a}}= & x_{2}^{T} M_{0} \dot{x}_{2}+\frac{1}{2} x_{2}^{T} \dot{M}_{0} x_{2}+x_{1}^{T} K \dot{x}_{1}=x_{2}^{T}\left(\Gamma+\Delta-C_{0} \dot{q}-G-M_{0} \ddot{q}_{d}\right. \\
& \left.+M_{0} K x_{2}-M_{0} K^{2} x_{1}\right)+\frac{1}{2} x_{2}^{T} \dot{M}_{0} x_{2}+x_{1}^{T} K\left(x_{2}-K x_{1}\right) \\
= & x_{2}^{T}\left(\Gamma+\Delta-C_{0}\left(x_{2}+\dot{q}_{d}-K x_{1}\right)-G-M_{0} \ddot{q}_{d}+M_{0} K x_{2}-M_{0} K^{2} x_{1}\right) \\
& +\frac{1}{2} x_{2}^{T} M_{0} x_{2}+x_{1}^{T} K\left(x_{2}-K x_{1}\right)
\end{aligned}
$$

Aplicando la propiedad expuesta por Khalil en [13] en la Ec. (15):

$$
\boldsymbol{x}_{2}^{T}\left(\dot{M}_{0}-2 C_{0}\right) x_{2}=0
$$

Se tiene:

$$
\begin{aligned}
\dot{V}_{\mathrm{a}}= & x_{2}^{T}\left(\Gamma+\Delta-C_{0}\left(\dot{q}_{d}-K x_{1}\right)-G-M_{0} \ddot{q}_{d}+M_{0} K x_{2}-M_{0} K^{2} x_{1}\right) \\
& +x_{1}^{T} K\left(x_{2}-K x_{1}\right)
\end{aligned}
$$

Con el fin de garantizar la estabilidad asintótica del sistema en lazo cerrado, se define la ley de control como:

$$
\Gamma=M_{0} \ddot{q}_{d}+C_{0} \dot{q}_{d}-\|\Delta\|-K x_{1}-M_{0} K x_{2}-G
$$

Se obtiene:

$$
\dot{V}_{\mathrm{a}}=-x_{2}^{T} C_{0} K x_{1}-x_{2}^{T} M_{0} K^{2} x_{1}-x_{2}^{T} K x_{1}-x_{2}^{T}\|\Delta\|+x_{2}^{T} \Delta+x_{1}^{T} K x_{2}-x_{1}^{T} K^{2} x_{1}
$$

Con el fin de determinar si la derivada de $V_{a}$ es definida negativa, se aplica la norma a unos sumandos de la Ec. (18), y aplicando las propiedades de norma de matrices [14], se tiene:

$$
\begin{aligned}
\dot{V}_{\mathrm{a}} & =-x_{2}^{T} C_{0} K x_{1}-x_{2}^{T} M_{0} K^{2} x_{1}-\left\|x_{2}^{T} K x_{1}\right\|-\left\|x_{2}^{T}\right\| \Delta\|\|+\left\|x_{2}^{T} \Delta\right\|+\left\|x_{1}^{T} K x_{2}\right\|-x_{1}^{T} K^{2} x_{1} \\
& \leq-x_{2}^{T} C_{0} K x_{1}-x_{2}^{T} M_{0} K^{2} x_{1}-\left\|x_{2}^{T}\right\|\left\|K x_{1}\right\|-\left\|x_{2}^{T}\right\|\|\Delta\|+\left\|x_{2}^{T}\right\|\|\Delta\|+\left\|x_{1}^{T}\right\|\left\|K x_{2}\right\|-x_{1}^{T} K^{2} x_{1} \\
& \leq-x_{2}^{T} C_{0} K x_{1}-x_{2}^{T} M_{0} K^{2} x_{1}-x_{1}^{T} K^{2} x_{1} \\
& \leq-\left(C_{0} K+M_{0} K^{2}\right)\left\|x_{2}^{T}\right\|\left\|x_{1}\right\|-K^{2}\left\|x_{1}\right\|^{2}<\odot
\end{aligned}
$$

Se puede observar que $\dot{V}_{a}$ es definida negativa, luego la ley de control definida en la Ec. (18) hace que el sistema sea globalmente asintóticamente estable según el teorema de estabilidad de Lyapunov [7]. 


\section{LEY DE CONTROL ROBUSTA}

En esta sección, se presenta una ley de control desarrollada por Spong [6] y aplicada a un robot SCARA [15]. Para esta estrategia de control, se retoma el modelo dinámico inverso del manipulador que se muestra en (1), y se hace un procedimiento aritmético que lleva a una nueva representación (Ec. (19)):

$$
M(q) \ddot{q}+C(q, \dot{q}) \dot{q}+G(q)=Y(q, \dot{q}, \ddot{q}) \pi
$$

Donde $\boldsymbol{\pi}$ es un vector constante $n$-dimensional de los parámetros del robot y $Y$ es una matriz, que es función de la posición, velocidad y aceleración de las articulaciones del robot. Con el fin de garantizar la estabilidad asintótica, se define la ley de control robusta como:

$$
\mu= \begin{cases}-\rho \frac{Y^{T} \sigma}{\left\|Y^{T} \sigma\right\|} & \text { si }\left\|Y^{T} \sigma\right\|>\varepsilon \\ -\rho \frac{Y^{T} \sigma}{\varepsilon} & \text { si }\left\|Y^{T} \sigma\right\| \leq \varepsilon\end{cases}
$$

Donde $\varepsilon>0$ y r es la cota de incertidumbre paramétrica, que se conoce a priori, teniendo así una cota para definir la incertidumbre paramétrica. La demostración detallada de esta estrategia de control, se puede observar en [6].

\section{DESCRIPCIÓN DEL ROBOT}

Los robots SCARA poseen una configuración planar que les confiere una gran sencillez desde el punto de vista cinemático, así como posibilidades notables en cuanto a velocidad y precisión en tareas de ensamblado (pick-and-place). En muchas ocasiones, su accionar es directo, sin incluir reductores en sus articulaciones, con el fin de aprovechar la rapidez del mecanismo necesaria en determinadas tareas. La Figura 1 muestra la arquitectura del robot.

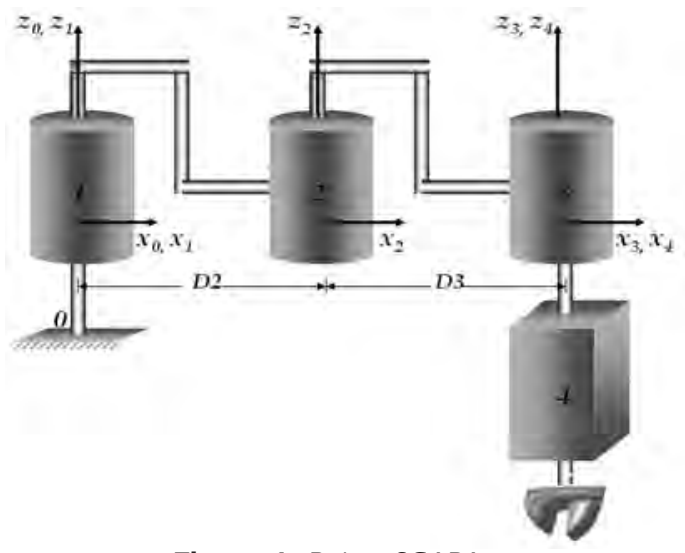

Figura 1. Robot SCARA 
La Tabla 1, muestra los parámetros geométricos del robot, siguiendo la parametrización de Khalil y Kleinfinger [13].

Tabla 1. Parámetros geométricos robot SCARA

\begin{tabular}{|c|c|c|c|c|c|}
\hline $\boldsymbol{J}$ & $\boldsymbol{\sigma}_{\boldsymbol{J}}$ & $\boldsymbol{\alpha}_{\boldsymbol{J}}$ & $\boldsymbol{d}_{\boldsymbol{J}}$ & $\boldsymbol{\theta}_{\boldsymbol{J}}$ & $\boldsymbol{r}_{\boldsymbol{J}}$ \\
\hline $\mathbf{1}$ & 0 & 0 & 0 & $\theta_{1}$ & 0 \\
\hline $\mathbf{2}$ & 0 & 0 & $\mathrm{D}_{2}$ & $\theta_{2}$ & 0 \\
\hline $\mathbf{3}$ & 0 & 0 & $\mathrm{D}_{3}$ & $\theta_{3}$ & 0 \\
\hline $\mathbf{4}$ & 1 & 0 & 0 & 0 & $\mathrm{r}_{4}$ \\
\hline
\end{tabular}

Se determinaron los parámetros de base y se agruparon por medio de la metodología propuesta por Khalil [13]; estos parámetros inerciales agrupados se muestran en la Tabla 2 [15].

Tabla 2. Parámetros dinámicos robot SCARA

\begin{tabular}{|c|c|c|c|}
\hline ZZG1 & 3.38 & MXG3 & $\mathbf{0 . 2}$ \\
\hline ZZG2 & 0.063 & MY2 & $\mathbf{0 . 0 0 1}$ \\
\hline ZZG3 & 0.1 & MYG3 & $\mathbf{0 . 1}$ \\
\hline MXG2 & $\mathbf{0 . 2 4 2}$ & la4 & $\mathbf{0 . 0 4 5}$ \\
\hline
\end{tabular}

Nota: Las unidades para los tensores de inercia son $\mathrm{Kg} \cdot \mathrm{m}^{2}$, para el primer momento de inercia $\mathrm{Kg} \cdot \mathrm{m}$, y para el motor de inercia $\mathrm{Kg} \cdot \mathrm{m}^{2}$.

\section{CONTROL POR PAR CALCULADO (CTC)}

En esta sesión, se desarrollará el control por desacoplamiento lineal o control por par calculado (Computed Torque Control - CTC), para el robot SCARA, que es una estrategia de control muy común utilizada en el control de posición de robots manipuladores $[13,16,17]$. La ley de control CTC se representa en la ecuación (21) y mayores detalles en [13].

$$
w(t)=\ddot{q}_{d}+K_{v}\left(\dot{q}_{d}-\dot{q}\right)+K_{p}\left(q_{d}-q\right)
$$

Donde $\boldsymbol{K}_{p}$ y $\boldsymbol{K}_{v}$ son matrices ( $\left.n \mathbf{x} n\right)$, diagonales y definidas positivas. Las constantes utilizadas para el control del manipulador se presentan en la Tabla 3, las cuales se obtuvieron mediante el bloque de Signal Constraint de Simulink ${ }^{\circledR}$, que se utilizó para sintonizar el controlador CTC. 
Tabla 3. Coeficientes de las matrices $K_{p}$ y $K_{v}$.

\begin{tabular}{|c|c|c|}
\hline ARTICULACIÓN & $\mathbf{K}_{\mathbf{p}}$ & $\mathbf{K}_{\mathbf{v}}$ \\
\hline $\mathbf{1}$ & 320000 & 1200 \\
\hline $\mathbf{2}$ & 280000 & 850 \\
\hline $\mathbf{3}$ & 200000 & 1200 \\
\hline $\mathbf{4}$ & 120000 & 700 \\
\hline
\end{tabular}

\section{RESULTADOS DE SIMULACIÓN}

En esta sección, se presentan los errores cartesianos obtenidos para el robot SCARA, utilizando el control robusto presentado por Spong, la estrategia backstepping con incertidumbre y el control CTC, para dos trayectorias, una circular y otra lineal con cambio de dirección (Figura 2). Estas trayectorias se realizaron en 5 y 3 segundos, con el fin de comparar el desempeño de las tres estrategias en los incrementos de velocidad.
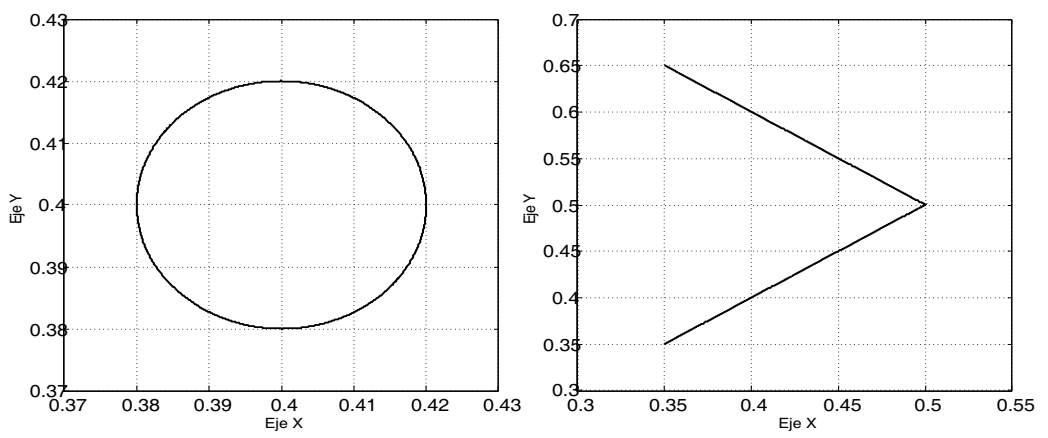

Figura 2. Trayectorias deseadas.

El proceso de sintonización del controlador propuesto por Spong se hace por medio de la selección de los valores de las matrices diagonales y definidas positivas $K$ y L, cuyos valores se muestran en la Tabla 4. Estas matrices se sintonizaron por medio del bloque Signal Constraintde Simulink $\mathbb{R}$.

Tabla 4. Coeficientes de las matrices $K y \mathrm{~L}$.

\begin{tabular}{|c|c|c|c|c|}
\hline Matrices & Art. 1 & Art. 2 & Art. 3 & Art. 4 \\
\hline$K$ & 1200 & 200 & 200 & 1500 \\
\hline$\Lambda$ & 1550 & 550 & 70 & 1500 \\
\hline
\end{tabular}

El proceso de sintonización del controlador backstepping se realiza por medio de la selección de los valores de la matriz diagonal y definida positiva $\boldsymbol{K}$. Dicha sintonización también se hizo por medio del bloque Signal Constraint, y se obtuvo como resultado, un valor de 800 para esta matriz. 
Las Figuras que se presentan a continuación, muestran el desempeño de los controladores robusto de Spong y backstepping, para las trayectorias mencionadas anteriormente, ejecutadas en un tiempo de 5 segundos. Luego se mostrarán los resultados globales de estos dos controladores, comparándolos con el más conocido control por par calculado.

La Figura 3 muestra el error cartesiano para las trayectorias deseadas de la estrategia de control robusto de Spong (Ec.(19)), para el modelo nominal del robot.

a)

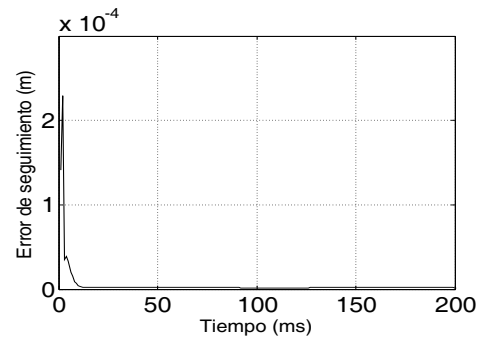

b)

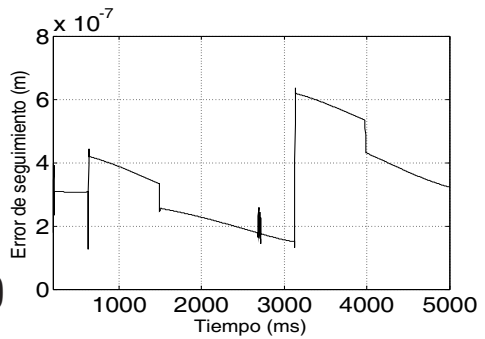

\section{c)}

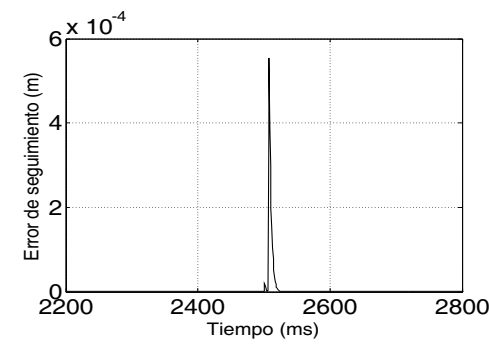

Figura 3. Error de seguimiento de la ley de control de Spong: a) estado transitorio trayectoria circular, b) estado estacionario trayectoria circular, c) cambio de dirección trayectoria lineal.

La Figura 4 muestra el error cartesiano para las trayectorias deseadas de la estrategia de control backstepping (Ec. (16)), para el modelo nominal de robot.

a)

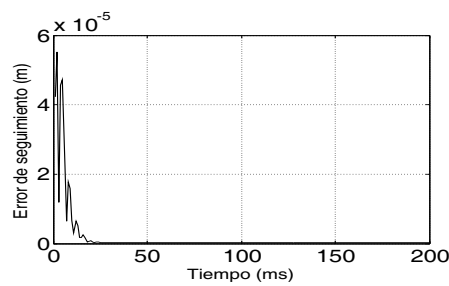

b)
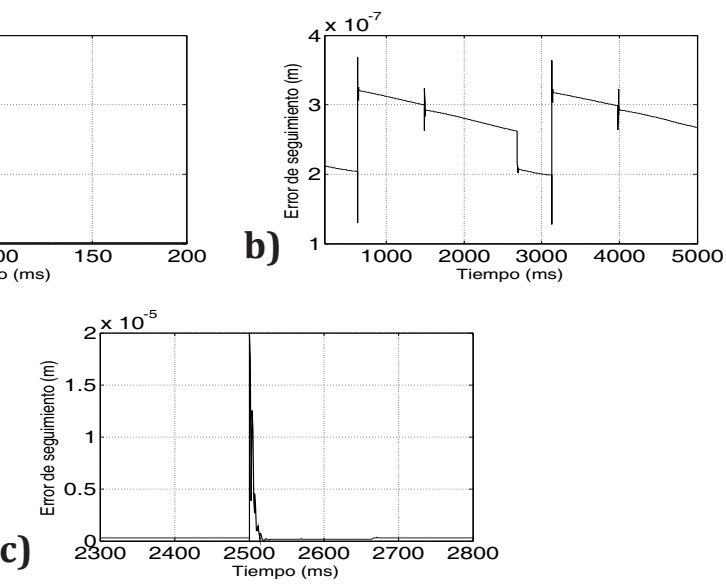

Figura 4. Error de seguimiento de la ley de control backstepping: a) estado transitorio trayectoria circular,

b) estado estacionario trayectoria circular, c) cambio de dirección trayectoria lineal 
Las Figuras 5 y 6 presentan los errores cartesianos, teniendo en cuenta una incertidumbre paramétrica del 10\%, para las estrategias de control robusto de Spong y backstepping respectivamente.

a)

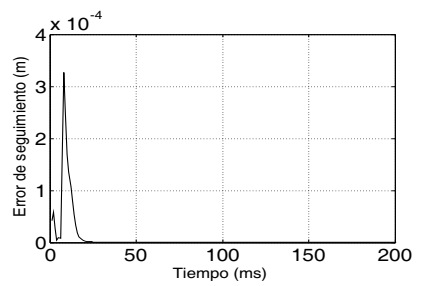

b)

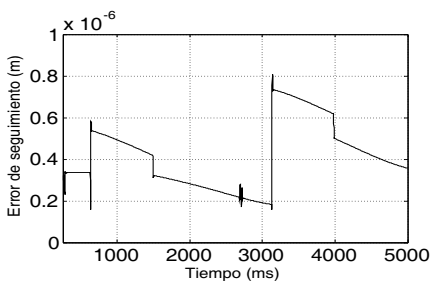

c)

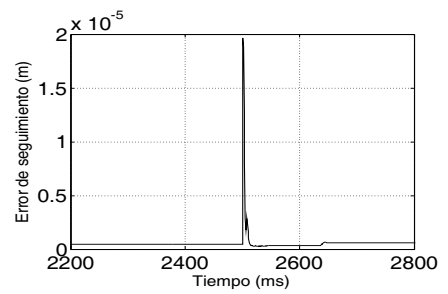

Figura 5. Error de seguimiento de la ley de control de Spong con incertidumbre del 10\%:a) estado transitorio trayectoria circular, b) estado estacionario trayectoria circular, c) cambio de dirección trayectoria lineal.

a)

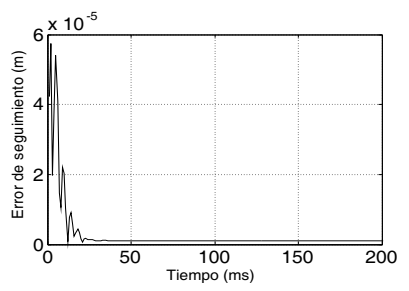

b)

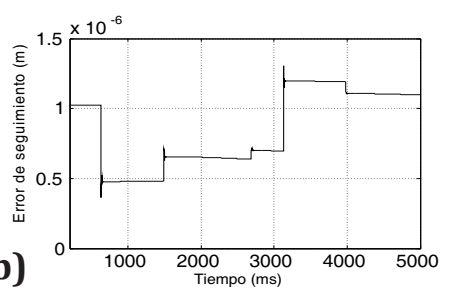

c)

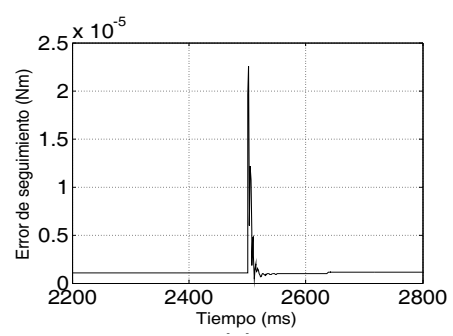

Figura 6. Error de seguimiento de la ley de control backstepping con incertidumbre del 10\%: a) estado transitorio trayectoria circular, b) estado estacionario trayectoria circular, c) cambio de dirección trayectoria lineal.

En la Tabla 5, se consignan los datos del error cartesiano teniendo en cuenta diferentes porcentajes de incertidumbre paramétrica, incluyendo el control por par calculado con el fin de observar el desempeño global de los tres controladores. 
Tabla 5. Error cartesiano para modelo con incertidumbre para un tiempo de ejecución de 5seg.

\begin{tabular}{|c|c|c|c|c|c|c|c|c|c|}
\hline \multirow{2}{*}{ 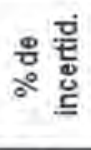 } & \multicolumn{3}{|c|}{ Error de Seguimiento CTC $(\mathrm{m})$} & \multicolumn{3}{|c|}{ Error de seguimiento CRS $(\mathrm{m})$} & \multicolumn{3}{|c|}{ Error de seguimiento $\mathrm{CBI}(\mathrm{m})$} \\
\hline & $\begin{array}{l}\text { Circ, } \\
\text { Trans. }\end{array}$ & $\begin{array}{l}\text { Circ. } \\
\text { Esta. }\end{array}$ & Lineal & $\begin{array}{l}\text { Circ. } \\
\text { Trans. }\end{array}$ & $\begin{array}{l}\text { Circ. } \\
\text { Esta. }\end{array}$ & Lineal & $\begin{array}{l}\text { Circ. } \\
\text { Trans. }\end{array}$ & $\begin{array}{l}\text { Circ. } \\
\text { Esta. }\end{array}$ & Lineal \\
\hline 0 & $3 \times 10^{-4}$ & $1 \times 10^{-6}$ & $2.7 \times 10^{-5}$ & $3.65 \times 10^{-4}$ & $6.4 \times 10^{-7}$ & $5.5 \times 10^{-4}$ & $5.5 \times 10^{-5}$ & $3.710^{-7}$ & $2 \times 10^{-5}$ \\
\hline 10 & $3.4 \times 10^{-4}$ & $1 \times 10^{-6}$ & $2.8 \times 10^{-5}$ & $1.8 \times 10^{-3}$ & $7.2 \times 10^{-7}$ & $2 \times 10^{-5}$ & $5.6 \times 10^{-5}$ & $8.1 \times 10^{-7}$ & $2 \times 10^{-5}$ \\
\hline-10 & $3.1 \times 10^{-4}$ & $1.1 \times 10^{-6}$ & $2.5 \times 10^{-5}$ & \multicolumn{2}{|c|}{ Inestable } & $2 \times 10^{-5}$ & $5.4 \times 10^{-5}$ & $7.2 \times 10^{-7}$ & $2 \times 10^{-5}$ \\
\hline 20 & $3.4 \times 10^{-4}$ & $9.5 \times 10^{-7}$ & $3 \times 10^{-5}$ & $1.7 \times 10^{-4}$ & $8 \times 10^{-7}$ & $2.1 \times 10^{-5}$ & $5.7 \times 10^{-5}$ & $1.3 \times 10^{-6}$ & $2.2 \times 10^{-5}$ \\
\hline-20 & $5.2 \times 10^{-5}$ & $1 \times 10^{-6}$ & $2.2 \times 10^{-5}$ & \multicolumn{3}{|c|}{ Inestable } & $5.1 \times 10^{-5}$ & $1 \times 10^{-6}$ & $2 \times 10^{-5}$ \\
\hline 40 & $1.7 \times 10^{-4}$ & $1 \times 10^{-6}$ & $3.1 \times 10^{-5}$ & $9.1 \times 10^{-4}$ & $1 \times 10^{-6}$ & $2.2 \times 10^{-5}$ & $2.7 \times 10^{5}$ & $2.2 \times 10^{-6}$ & $25 \times 10^{-5}$ \\
\hline-40 & \multicolumn{3}{|c|}{ Inestable } & \multicolumn{3}{|c|}{ Inestable } & \multicolumn{3}{|c|}{ Inestable } \\
\hline 60 & $1.7 \times 10^{-4}$ & $1.2 \times 10^{-6}$ & $3.6 \times 10^{-5}$ & $1.41 \times 10^{-3}$ & $1.2 \times 10^{-6}$ & $2.2 \times 10^{-4}$ & $7.8 \times 10^{-5}$ & $3.2 \times 10^{-6}$ & $2.7 \times 10^{-5}$ \\
\hline-60 & \multicolumn{3}{|c|}{ Inestable } & \multicolumn{3}{|c|}{ Inestable } & \multicolumn{3}{|c|}{ Inestable } \\
\hline 100 & $1.7 \times 10^{-4}$ & $1.3 \times 10^{-6}$ & $4.3 \times 10^{-5}$ & $1.7 \times 10^{-3}$ & $1.8 \times 10^{-6}$ & $3 \times 10^{-5}$ & $21 \times 10^{-4}$ & $5.2 \times 10^{-8}$ & $3.2 \times 10^{-5}$ \\
\hline
\end{tabular}

CTC: Control por par calculado, CRS: Control robusto de Spong,

CBI: Control backstepping con incertidumbre.

En la figura 7, se evidencia el error de seguimiento, estado transitorio de las trayectorias lineal y circular de la estrategia de control backstepping; la línea continua representa la trayectoria deseada y la línea punteada la realizada por el robot.

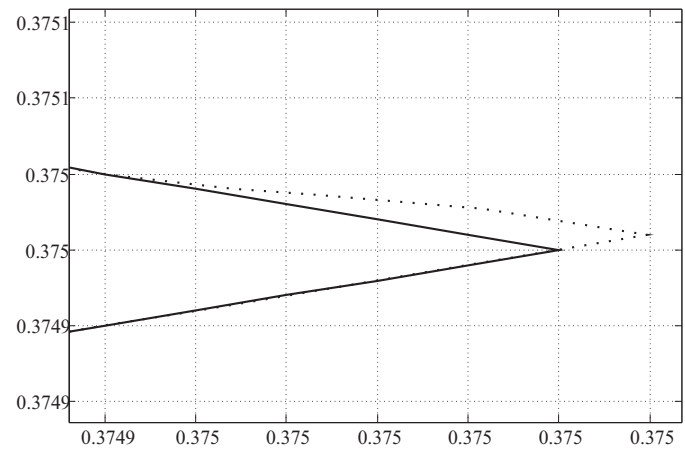

a)

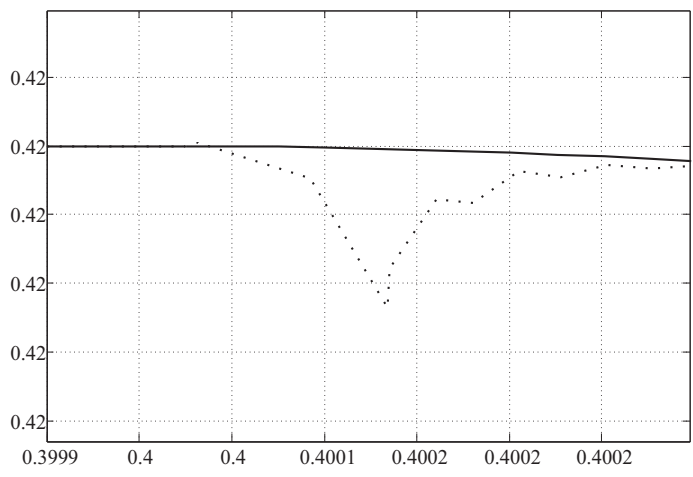

b)

Figura 7. Error de seguimiento, a) trayectoria lineal b) trayectoria circular. Línea seguida trayectoria deseada, línea punteada trayectoria realizada por el robot.

En términos generales los resultados obtenidos muestran un mejor desempeño de la ley CBI frente a los otros dos controladores. La inestabilidad de las estrategias de control CTC y CRS para una incertidumbre de $-40 \%$, se debe a que el modelo dista mucho del empleado en la sintonización de las leyes de control. Otros resultados importantes para resaltar, son los esfuerzos 
de control, que se ilustran a continuación, para el cambio de dirección de la consigna lineal. Las Figuras 8 y 9 son la respuesta del control propuesto por Spong y el control backstepping para el manipulador nominal, y con un 10\% de incertidumbre respectivamente.

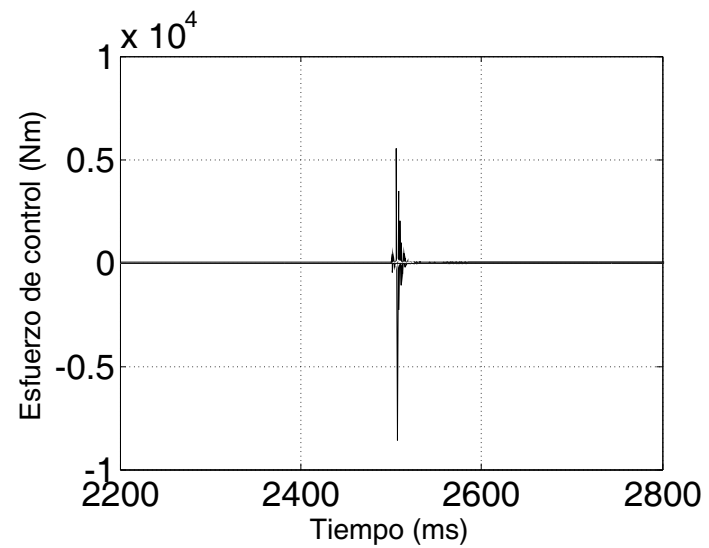

a)

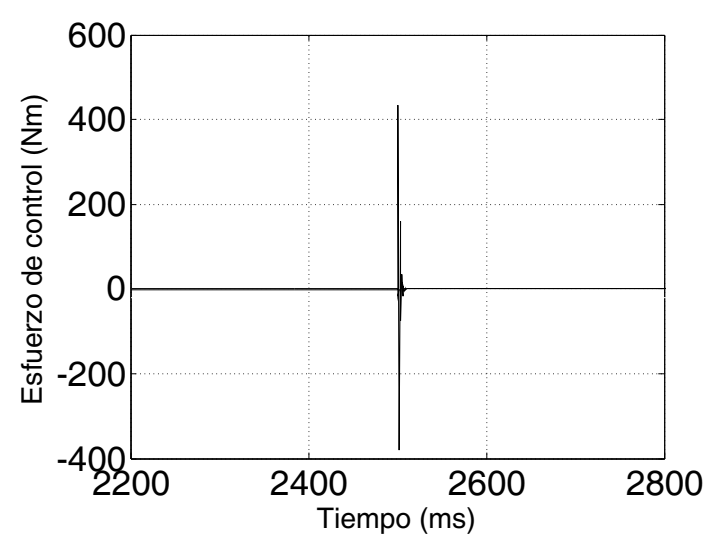

b)

Figura 8. Esfuerzos de control en el cambio de dirección del controlador de Spong: a) modelo nominal, b) modelo con incertidumbre del $10 \%$.

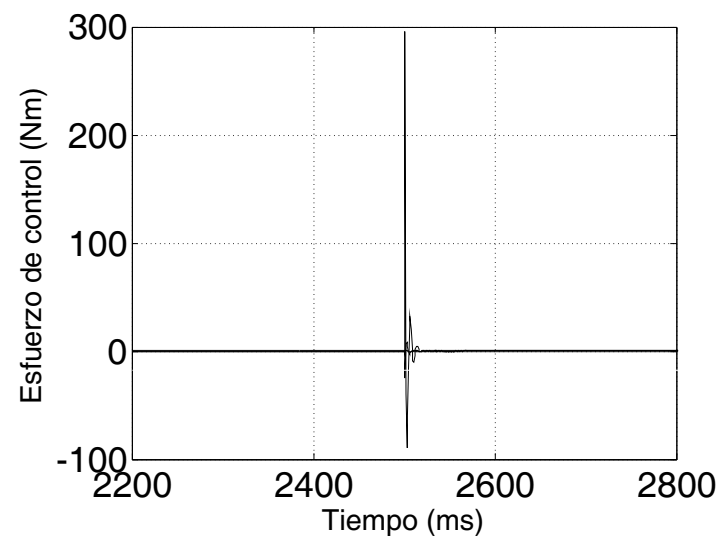

a)

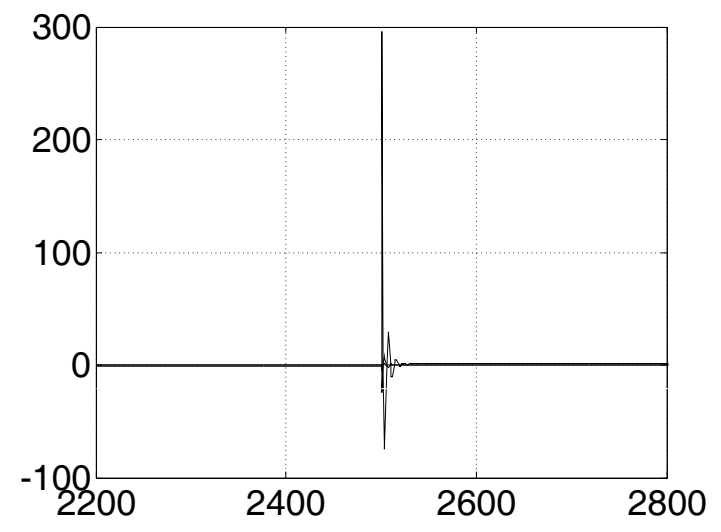

b)

Figura 9. Esfuerzos de control en el cambio de dirección controlador backstepping: al modelo nominal, b) modelo con incertidumbre del $10 \%$.

La Tabla 6 muestra los datos de los esfuerzos pico de control, producidos por las tres estrategias de control presentadas en este artículo, para diferentes porcentajes de incertidumbre y un tiempo de ejecución de las trayectorias de 5 segundos. 
Tabla 6. Esfuerzos de control para un tiempo de ejecución de 5 seg

\begin{tabular}{|c|c|c|c|c|c|c|c|c|c|}
\hline \multirow{2}{*}{ 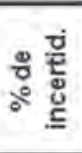 } & \multicolumn{3}{|c|}{$\begin{array}{l}\text { Esfuerzo de control CTC } \\
(\mathrm{Nm})\end{array}$} & \multicolumn{3}{|c|}{ Esfuerzo de control CRS (Nm) } & \multicolumn{3}{|c|}{ Esfuerzo de control CBI (Nm) } \\
\hline & \begin{tabular}{l|} 
Circ. \\
Trans.
\end{tabular} & $\begin{array}{l}\text { Circ. } \\
\text { Esta. }\end{array}$ & Lineal & Circ. Trans. & & Lineal & $\begin{array}{l}\text { Circ. } \\
\text { Trans. }\end{array}$ & $\begin{array}{l}\text { Circ. } \\
\text { Esta. }\end{array}$ & Lineal \\
\hline 0 & 540,340 & $6,-4$ & -60 & $300,-8600$ & $1.6,-16$ & $5500,-8700$ & $500,-650$ & $1.3,-17$ & $00 .-736$ \\
\hline 10 & $0 .-300$ & $5,-4$ & -60 & $000,-23500$ & $24,-21$ & $430,-380$ & $500,-740$ & -21 & 0.74 \\
\hline-10 & $40,-410$ & $8,-4$ & $60,-60$ & \multicolumn{2}{|c|}{ Inestable } & $540,-580$ & $510,-590$ & $11,-14$ & $300,-100$ \\
\hline 20 & $550,-570$ & $5,-4$ & $60,-60$ & $27800,-18500$ & $1.9,-25$ & $430,-300$ & $450,-810$ & $1.5,-25$ & $300,-74$ \\
\hline-20 & $300,-220$ & $10,-10$ & $60,-60$ & \multicolumn{3}{|c|}{ Inestable } & $550,-520$ & $0.9,-11$ & $300,-100$ \\
\hline 40 & $0,-380$ & $5,-9$ & $60 .-60$ & $11200,-6800$ & $17 .-34$ & & $450,-1100$ & $17,-34$ & $300,-69$ \\
\hline-40 & \multicolumn{3}{|c|}{ Inestable } & \multicolumn{3}{|c|}{ Inestable } & \multicolumn{3}{|c|}{ Inestable } \\
\hline 60 & $60,-330$ & $45,-15$ & $60,-60$ & $16500,-17000$ & $1.7 .-45$ & $2800,-2350$ & $580,-1380$ & $2,-45$ & $300,-70$ \\
\hline-60 & \multicolumn{3}{|c|}{ Inestable } & \multicolumn{3}{|c|}{ Inestable } & \multicolumn{3}{|c|}{ Inestable } \\
\hline 100 & $250,-150$ & $4,3,-29$ & $60,-60$ & $11500,-21600$ & $2.3,-70$ & $432,-70$ & $1340,-3400$ & $3,-70$ & $300,-80$ \\
\hline
\end{tabular}

Las Tablas 7 y 8 presentan la respuesta de las estrategias de control frente a las trayectorias deseadas para un tiempo de ejecución de 3 segundos y los esfuerzos de control para este incremento de velocidad. Se puede observar que al solicitarse una consigna más rápida, el control $\mathrm{CBI}$ reacciona de mejor manera y con unos esfuerzos de control globalmente menores.

Tabla 7. Error cartesiano para un tiempo de ejecución de 3 seg

\begin{tabular}{|c|c|c|c|c|c|c|c|c|c|}
\hline \multirow{2}{*}{ 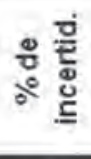 } & \multicolumn{3}{|c|}{ Error de Seguimiento CTC (m) } & \multicolumn{3}{|c|}{ Error de seguimiento CRS (m) } & \multicolumn{3}{|c|}{ Error de seguimiento $\mathrm{CBI}(\mathrm{m})$} \\
\hline & $\begin{array}{c}\text { Circ. } \\
\text { Trans. } \\
\end{array}$ & $\begin{array}{l}\text { Circ. } \\
\text { Esta. }\end{array}$ & Lineal & $\begin{array}{l}\text { Circ. } \\
\text { Trans. }\end{array}$ & $\begin{array}{l}\text { Circ. } \\
\text { Esta. }\end{array}$ & Lineal & $\begin{array}{l}\text { Circ. } \\
\text { Trans. }\end{array}$ & $\begin{array}{l}\text { Circ. } \\
\text { Esta. }\end{array}$ & Lineal \\
\hline 0 & $14 \times 10^{-3}$ & $2 \times 10^{-6}$ & $2.5 \times 10^{-4}$ & $2.5 \times 10^{-4}$ & $8 \times 10^{-7}$ & $2 \times 10^{-5}$ & $8.4 \times 10^{-5}$ & $3.610^{-7}$ & $2 \times 10^{-5}$ \\
\hline 10 & $1.2 \times 10^{-3}$ & $2 \times 10^{-6}$ & $2.6 \times 10^{-4}$ & $6.2 \times 10^{-4}$ & $9 \times 10^{-6}$ & $2 \times 10^{-5}$ & $8.5 \times 10^{-5}$ & $9.4 \times 10^{-7}$ & $2 \times 10^{-5}$ \\
\hline-10 & $5.1 \times 10^{-3}$ & $2 \times 10^{-8}$ & $2.2 \times 10^{-4}$ & \multicolumn{2}{|c|}{ Inestable } & $2 \times 10^{-5}$ & $7.9 \times 10^{-5}$ & $6.8 \times 10^{-7}$ & $2 \times 10^{-5}$ \\
\hline 20 & $4.2 \times 10^{-3}$ & $2 \times 10^{-6}$ & $2.6 \times 10^{-4}$ & $3.3 \times 10^{-3}$ & $1 \times 10^{-6}$ & $1.9 \times 10^{-5}$ & $8.7 \times 10^{-5}$ & $1.45 \times 10^{-6}$ & $2.2 \times 10^{-5}$ \\
\hline-20 & $5 \times 10^{-3}$ & $2 \times 10^{-6}$ & $2 \times 10^{-4}$ & \multicolumn{3}{|c|}{ Inestable } & $7.9 \times 10^{-5}$ & $1 \times 10^{-6}$ & $2 \times 10^{-5}$ \\
\hline 40 & $2.5 \times 10^{-4}$ & $2.1 \times 10^{-6}$ & $3 \times 10^{-4}$ & $16.5 \times 10^{-4}$ & $1.4 \times 10^{-6}$ & $2.8 \times 10^{-5}$ & $4.6 \times 10^{-5}$ & $2.4 \times 10^{-6}$ & $2.5 \times 10^{-5}$ \\
\hline-40 & \multicolumn{3}{|c|}{ Inestable } & \multicolumn{3}{|c|}{ Inestable } & \multicolumn{3}{|c|}{ Inestable } \\
\hline 60 & $3.5 \times 10^{-3}$ & $2.3 \times 10^{-6}$ & $3 \times 10^{-4}$ & $1.05 \times 10^{-3}$ & $1.7 \times 10^{-6}$ & $2.6 \times 10^{-5}$ & $5.4 \times 10^{-5}$ & $3.5 \times 10^{-6}$ & $2.7 \times 10^{-5}$ \\
\hline-60 & \multicolumn{3}{|c|}{ Inestable } & \multicolumn{3}{|c|}{ Inestable } & \multicolumn{3}{|c|}{ Inestable } \\
\hline 100 & $8 \times 10^{-4}$ & $3 \times 10^{-6}$ & $3.9 \times 10^{-4}$ & $1.7 \times 10^{-3}$ & $2.6 \times 10^{-6}$ & $3.1 \times 10^{-5}$ & $3.6 \times 10^{-4}$ & $5.5 \times 10^{-6}$ & $3.2 \times 10^{-5}$ \\
\hline
\end{tabular}


Tabla 8. Esfuerzos de control para un tiempo de ejecución de 3 seg

\begin{tabular}{|c|c|c|c|c|c|c|c|c|c|}
\hline \multirow{2}{*}{ 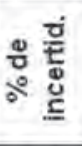 } & \multicolumn{3}{|c|}{ Esfuerzo de control CTC (Nm) } & \multicolumn{3}{|c|}{ Esfuerzo de control CRS ( $\mathrm{Nm}$ ) } & \multicolumn{3}{|c|}{ Esfuerzo de control CBI (Nm) } \\
\hline & Circ. Trans. & $\begin{array}{l}\text { Circ. } \\
\text { Esta. }\end{array}$ & Lineal & Circ. Trans. & $\begin{array}{l}\text { Circ. } \\
\text { Esta. }\end{array}$ & Lineal & $\begin{array}{c}\text { Circ. } \\
\text { Trans. }\end{array}$ & $\begin{array}{l}\text { Circ. } \\
\text { Esta. }\end{array}$ & Lineal \\
\hline 0 & $10000,-7500$ & $9.5 .-9.5$ & $700,-900$ & $9500,-7500$ & $14,-17.5$ & $420,-430$ & $560,-940$ & $1,7,-17$ & $300,-90$ \\
\hline 10 & $9000,-7500$ & $7,-7$ & $750,-900$ & $18000,-10000$ & $18,-22$ & $420,-380$ & $500,-1000$ & $2,-21$ & $300,-75$ \\
\hline-10 & $17000,-12000$ & $13,-13$ & $750,-900$ & \multicolumn{2}{|c|}{ Inestable } & $550,-580$ & $620,-850$ & 1.6. -14 & $300,-100$ \\
\hline 20 & $1900,-1900$ & $7,-7$ & $750,-900$ & $65000,-45000$ & $1.7,-25$ & $420,-300$ & $460,-1100$ & $2.1,-25$ & $300,-75$ \\
\hline-20 & $4500,-3200$ & $15,-15$ & $750,-900$ & \multicolumn{3}{|c|}{ Inestable } & $710,-770$ & $1.3,-11$ & $300,-130$ \\
\hline 40 & $1500,-1500$ & $6.5,-9$ & $750,-900$ & $20000,-15000$ & $2.1,-35$ & $420,-180$ & $400,-1250$ & $2.6,-34$ & $300,-69$ \\
\hline-40 & \multicolumn{3}{|c|}{ Inestable } & \multicolumn{3}{|c|}{ inestable } & \multicolumn{3}{|c|}{ Inestable } \\
\hline 60 & $6200_{n}-6200$ & $6.2,-15$ & $750,-900$ & $14000,-14000$ & $22,-45$ & $430,-90$ & $400,-1510$ & $3,-45$ & $300,-70$ \\
\hline-60 & \multicolumn{3}{|c|}{ Inestable } & \multicolumn{3}{|c|}{ Inestable } & \multicolumn{3}{|c|}{ Inestable } \\
\hline 100 & $2200,-2200$ & $5.2,-28$ & $750,-900$ & $20000,-26000$ & $3.5,-70$ & $430 .-70$ & $630,-2400$ & $43-70$ & $300,-80$ \\
\hline
\end{tabular}

\section{CONCLUSIONES}

Las leyes de control por modelo de referencia utilizadas sobre manipuladores robóticos adolecen del hecho de que las dinámicas no modeladas, la incertidumbre paramétrica y el acoplamiento dinámico afectan, fuertemente su desempeño.

La obtención de una ley de control que involucre la incertidumbre paramétrica y que presente un buen desempeño para el seguimiento de trayectorias como la que se desarrolló en este trabajo (backstepping), muestra que es posible generar un sistema controlado robusto sin tener grandes esfuerzos de control. Además, este tipo de controlador mantiene un buen desempeño al incrementar la velocidad de operación del manipulador. A pesar del trabajo adicional que representa el diseño y aplicación de esta ley, en aplicaciones industriales que requieran buena velocidad y el modelo matemático del robot no está muy bien definido, este tipo de estrategia puede proporcionar una buena solución.

Futuros trabajos realizarán una comparación de este controlador con otras técnicas avanzadas de control, antes de proceder a su implementación sobre un robot real.

\section{AGRADECIMIENTOS}

Los autores agradecen a la Universidad del Cauca por el apoyo en el desarrollo de este proyecto. 


\section{REFERENCIAS BIBLIOGRÁFICAS}

[1] Sage H., Mathelin M., and Ostertag E., (1999). Robust control of robot manipulators: a survey. En: International Journal of Control. Vol. 72 (16), pp. 1498 - 1522.

[2] Burkan R., and Uzmay I., (2003). Variable upper bounding approach for adaptive-robust control in robot control. En: Journal of Intelligent and Robotic Systems. Vol. 37 (4), pp. 427-442.

[3] Burkan R., and Uzmay I., (2005). Logarithmic based robust control approach to parametric uncertainty for control of robot manipulators, En: Intenational Journal of Robust and Nonlinear Control. Vol. 15 (10), pp. 427 - 436.

[4] Mnif F., (2004). A mixed optimal/robust control for robot manipulators, En: Systems and Control Engineering. Vol. 218 (4), pp. 311-321.

[5] Alonge F., D'Ippolito and Raimondi F. (2004). Globally convergent adaptive and robust control of robotic manipulators for trajectory tracking, En: Control Engineering Practice. Vol. 12 (9), pp. 1091-1100.

[6] Spong M., (1992). On the robust control of robot manipulators. En: IEEE Transaction on Automatic Control. Vol. 37 (11), pp. 668-672.

[7] KrstícM., Sun J., and Kokotovíc V., (1996). Robust control of nonlinear systems with inputunmodeled dynamics. En: IEEE Transaction on Automatic Control. Vol. 41 (6), pp. 913-920.

[8] Lotfatar A., and Eghtesad M., (2007). Applicationand comparison of passivity-based and integrator backstepping control methods for trajectory tracking of rigid-link robot manipulators incorporating motor dynamics. En: International Journal of robotics and Automation. Vol. 22 (3).

[9] LU C., Hwang Y., and Shen Y., (2010). Backstepping sliding mode control for a pneumatic control system. En: Proceedings of the Intitution of Mechanical Engineer. 763 p.

[10] Yao J., Zhu X., and Zhou Z., (2008). The design of slading mode control system based on backstepping theory for BTT UAV. Control and Intelligent system. Vol. 36 (4), pp. 347-354.

[1 1] Hemici B., Nezli L., Tadjine M., and Boucherit M., (2006). Robust PID/Backsteppineg control design for permanent magnet synchronous motor drive. En: Control and Inteligent Systems. Vol. 34 (3), pp. 194-200.

[12] Härkegard O., (2001). Flight control design using backstepping. Ph.D. Thesis, Sweden: Linköping Studies in Science and Technology. 
[13] Khalil W., and Dombe E., (2002). Modeling, identification and control of robots. London: Hermes Penton Science. pp. 347-376.

[14] Chen C., (1999). Linear system theory and desing. Oxford University Press, pp. 77-79.

[15] Caraflore G., Indri M., and Bona B., (2001). Robot dynamic calibration: Optimal excitation trajectories and experimental parameter estimation. En: Journal of Robotic Systems. Vol. 18 (2), pp. 55-68.

[16] Mosquera V., and Vivas A., (2006). Robust control for a SCARA robot with parametric uncertainty. En: Proceedings of the International control conference, Glagow.

[17] Mosquera V., (2009). Control robusto de un robot de más de 3 grados de libertad. Tesis de Maestría. Universidad del Cauca, Popayán. 